\title{
ANTIMICROBIAL ACTIVITY OF ANTHOCEPLALUS CADAMBA AND SCIRPUS KYSOOR ROXB. AGAINST FOOD PATHOGENS
}

\author{
HEMANGI DATAR ${ }^{*}$, AJIT DATAR \\ Guru Nanak Institute of Research and Development, Guru Nanak Khalsa College, Mumbai, Maharashtra, India \\ Email: hemangi131@gmail.com
}

Received: 18 Jul 2016, Revised and Accepted: 20 Aug 2016

\section{ABSTRACT}

Objective: Aim of the present study was to evaluate the antimicrobial activity of ripened and unripened fruits of Anthocephalus cadamba and Scirpus kysoor Roxb. tuber against food pathogens.

Methods: Different solvent extracts of Anthocephalus cadamba fruits and Scirpus kysoor Roxb. tubers were screened for its antimicrobial activity against Gram negative and Gram positive bacterial cultures by agar well diffusion method as well as by minimum inhibitory concentration (MIC) and minimum bactericidal concentration (MBC). MIC was carried out by broth microdilution method.

Results: All the plant materials exhibit antimicrobial activity. Among all plant materials, macerated ethyl acetate extract of Scirpus kysoor Roxb. tuber showed lowest MIC against Bacillus Cereus $0.312 \mathrm{mg} / \mathrm{ml}$. In the case of ripened Anthocephalus cadamba fruits, macerated ethanol extract was found to be more potent whereas in the case of unripened Anthocephalus cadamba fruit macerated ethyl acetate extract was found to be more active on the basis of MIC.

Conclusion: This study revealed that all the plant materials possess significant antimicrobial activity against food pathogens hence may be used as a source of safe herbal antimicrobial agent.

Keywords: Anthocephalus cadamba, Scirpus kysoor Roxb., Antimicrobial activity, MIC, MBC

(C) 2016 The Authors. Published by Innovare Academic Sciences Pvt Ltd. This is an open access article under the CC BY license (http://creativecommonsorg/licenses/by/4.0/] DOI: http://dx.doi.org/10.22159/ijcpr.2016v8i4.15269

\section{INTRODUCTION}

In recent years the main concern of regulatory and food industries is an increase in foodborne illness outbreaks by pathogens throughout the world. The other concerns are the increasing antibiotic resistance of some pathogens associated with foodborne illness and side effects of synthetic drugs. Hence it is essential to discover newer drugs with no or lesser microbial resistance [1].

Since ancient times plants have been utilized as therapeutic agents for various kinds of illness. Some natural substances originating from plants exhibit antibacterial properties [2]. Because of their lesser side effects and low resistance to pathogens, they are more popular than synthetic drugs. Because of such antibacterial properties, plant materials have been even used for prevention of food spoilage and preservation of foods from centuries. The antibacterial properties of plants are because of the secondary metabolites like alkaloids, terpenes, tannins, phenols, etc [3].

Anthocephalus cadamba and Scirpus kysoor Roxb. are two medicinally important plants containing various secondary metabolites. Anthocephalus cadamba belongs to family Rubiaceae. It's common name is Kadam,in Marathi, it is known as Kadamba. Phytochemical investigation of different parts of Anthocephalus cadamba showed the presence of chemical constituents such as indole alkaloids, terpenoids, saponins, sapogenins, terpenes, steroids, fats, reducing sugar, glycosides and flavonoids [4]. Literature survey revealed that various parts of Anthocephalus cadamba possess antimicrobial activity [5-8]. The fruits showed significant antibacterial activity against some bacteria. But its antimicrobial activity against food-borne pathogens has been not yet reported.

Kaseru (Scirpus kysoor) belongs to family Cyperaceae. Its common name is Water chestnut and commonly it is found on the margins of ponds and swampy places throughout India. It is a good source of progesterone, starch and sugar. Various medicinal properties of Scirpus kysoor Roxb. has been described in Ayurveda [9, 10]. Antimicrobial property of Scirpus kysoor Roxb. has not been reported till date. Hence this study was carried out for exploring the antimicrobial potential of both these plants against food pathogens.

\section{MATERIALS AND METHODS}

All the chemicals reagents were procured from LobaChemie, India Pvt. Ltd. and microbiological media were obtained from Hi-media Laboratories.

\section{Collection and authentication of the plant material}

Anthocephalus cadamba fruits (ripened and unripened) were collected from Kaylan (Maharashtra) and Scirpus kysoor Roxb. tubers were collected from western ghats of Maharashtra. All these materials were authenticated from Agharkar Research Institute, Pune.

\section{Extraction of plant material}

Plant materials were shade dried, kept in an oven at $37^{\circ} \mathrm{C}$ and finally ground to fine powder. Fine powder of Scirpus kysoor Roxb. (sieved through sieve of mesh no.44) and coarse powder of Anthocephalus cadamba fruit was used for extraction. All the 3 materials were extracted by Kinetic Maceration method on a rotary shaker for $24 \mathrm{~h}$ using solvents of different polarities i.e. Methanol, Ethanol, Ethyl Acetate, Chloroform, pet ether and Hexane [10, 11].

On the basis of results of antimicrobial activity (Zone of Inhibition) shown by macerated extracts of above solvents, 2 solvents were chosen for soxhlet extraction $[11,12]$ for each material.

For Ripened Anthocephalus cadamba fruits Methanol and Ethyl acetate, for unripened Anthocephalus cadamba fruits Ethanol and Methanol and for Scirpus kysoor Roxb tubers. Ethyl acetate and pet ethers were chosen as solvents for soxhlet extraction.

\section{Culture and maintenance of microorganisms}

Staphylococcus aureus (S. aureus) ATCC 25922 and Bacillus cereus (B. cereus) ATCC 11778 were 2-gram positive bacteria and Escherichia coli (E. coli) ATCC8739, Salmonella abony (S. abony) ATCC 6017 and 
Shigella boydii (S. boydii) ATCC 8700 were 3-gram negative bacteria used for this assay. All these bacterial cultures were obtained from Guru Nanak Institute of Research and Development (GNIRD) and Microbiology Department of G. N. Khalsa College, Matunga. All the bacterial cultures were maintained on nutrient agar medium at $4{ }^{\circ} \mathrm{C}$ till further use.

\section{Preparation of inoculum}

A single colony was transferred in sterile $50 \mathrm{ml}$ of nutrient broth and incubated at $37^{\circ} \mathrm{C}$ for $24 \mathrm{~h}$. The concentration of bacterial cells was optimized to $0.5 \mathrm{McF}$ arland standard (that corresponds to $1.5 \mathrm{X} 10^{8}$ $\mathrm{CFU} / \mathrm{ml}$ ) at $620 \mathrm{~nm}$ for agar well diffusion assay.

\section{Antimicrobial bioassay}

Antimicrobial activity of all the materials was primarily evaluated by agar well diffusion method and further quantification was done by Minimum Inhibitory concentration (MIC) and Minimum Bactericidal Concentration (MBC) against the selected 5 food pathogens.

\section{Agar well diffusion method}

Agar well diffusion method was carried out as per guidelines provided by CLSI [13].

One ml of inoculum prepared was mixed thoroughly into $20 \mathrm{ml}$ of sterile nutrient agar and poured into Petri plate of $9 \mathrm{~cm}$ diameter. After solidification of agar, wells were made by using $8 \mathrm{~mm}$ of cork borer. Fifty $\mu \mathrm{l}$ of respective plant extracts were added in respective wells. Plates were incubated at $37^{\circ} \mathrm{C}$ for $24 \mathrm{~h}$ and zone of inhibition was measured in $\mathrm{mm}$. Dimethyl Sulphoxide (DMSO) was used as negative control. Ciprofloxacin (5 ppm) was used as positive control [7, 14].

All experiments were repeated three times independently, and the mean and standard deviation of the diameter of inhibition zones were calculated. Values obtained were expressed as mean \pm SD.

\section{Determination of minimum inhibitory concentration (MIC)}

The minimum inhibitory concentration (MIC) is defined as the lowest concentration of the antimicrobial agent that will inhibit the visible growth of a microorganism after overnight incubation $[15,16]$.

Two-fold microdilution broth method was used to determinate the MIC value [17].

A stock solution of 4 most active extracts of both the Anthocephalus cadamba fruits was serially diluted in 96-wells microtiter plate with Nutrient broth to obtain a concentration of 25, 12.5, 6.25,3.12, 1.56, 0.78 $\mathrm{mg} / \mathrm{ml}$ and that of Scirpus kysoor Roxb. tuber from10.0, 5.0, 2.5, 1.25, $0.625,0.312 \mathrm{mg} / \mathrm{ml}$. A standardized inoculum for each bacterial strain was prepared so as to give an inoculum size of approximately $5 \times 105$ $\mathrm{CFU} / \mathrm{ml}$ in each well. Positive control well contained inoculated growth medium without test samples. The negative control well included uninoculated medium and DMSO. .Microtiter plates were then kept at 37 ${ }^{\circ} \mathrm{C}$ for an overnight incubation. The MICs of each extract were determined after incubation following the addition of $40 \mu \mathrm{l} \mathrm{p}$ iodonitrotetrazolium salt(INT dye) and incubation at room temperature for $30 \mathrm{~min}$. Viable bacteria reduced this yellow dye to pink. The MIC of each sample was defined as its lowest concentration that prevented this change and then resulted in the complete inhibition of microbial growth $[18,19]$.

\section{Determination of minimum bactericidal concentration (MBC)}

Bactericidal Concentration (MBC) was determined by streaking samples from the wells with concentrations of MIC and above the MIC on new plates of Nutrient Agar. The MBC was considered as the lowest concentration of the extract associated with no bacterial culture that is indicating $99.5 \%$ killing of the original inoculum [20].

\section{RESULTS AND DISCUSSION}

\section{Antimicrobial bioassay}

In the present investigation, the antimicrobial activity of different extracts of unripened and ripened Anthocephalus cadamba fruits and tuber of Scirpus Kysoor Roxb. were evaluated against five food pathogens. The antimicrobial activity was determined using agar well diffusion method and microdilution method summarized in table 3-8. The activity was quantitatively assessed on the basis of minimum inhibitory concentration (MIC), minimum bactericidal concentration (MBC) and ratio of MIC and MBC.

Measurement of antimicrobial activity using agar well diffusion method

The results of Agar Well Diffusion are summarized in table 1-3 which revealed that most of the plant extracts showed antibacterial activity at various degrees ranging from 10-24 mm (except n-hexane extract of the unripened Anthocephalus cadamba for E. coli).

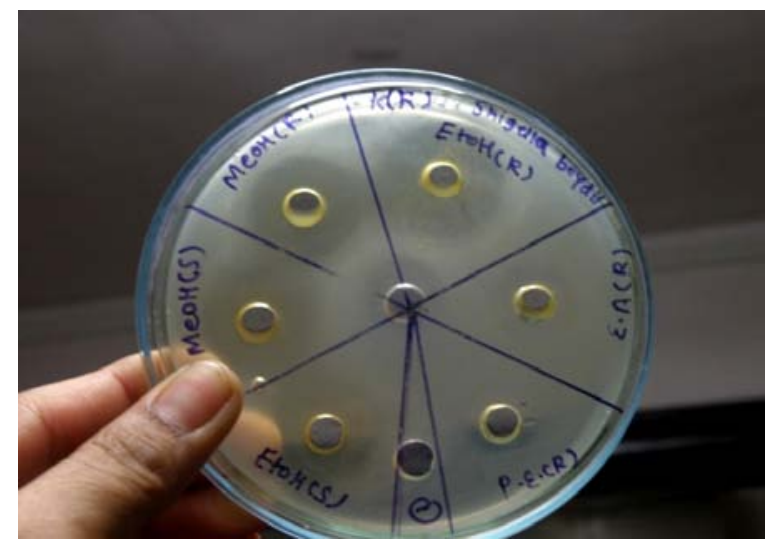

Fig. 1: Ripened Anthocephalus cadamba fruit Zone of Inhibition against Shigella boydii, EtoH(R)-macerated ethanolic extract EtoH(s)-soxhlet ethanolic extract, $\operatorname{MeoH}(R)$-macerated methanolic extract MeoH(s)-soxhlet methanolic extract, E. A.(R)-macerated ethyl acetate extract $P$. E.(R)-macerated pet ether extract, of ripened cadamba fruit '-'-negative control and center well-positive control

Table 1: Zone of inhibition of ripened Anthocephalus Cadamba (mm)

\begin{tabular}{llllc}
\hline $\begin{array}{l}\text { Ripened Anthocephalus } \\
\text { Cadamba }\end{array}$ & Staphylococcus aureus & Bacillus cereus & Escherichia coli & Salmonella abony \\
\hline Methanol & $16.0 \pm 1.00$ & $15.0 \pm 1.00$ & $16.33 \pm 0.57$ & $10.0 \pm 1.00$ \\
bthanol & $18.0 \pm 0.00$ & $18.0 \pm 0.00$ & $17.0 \pm 1.00$ & $19.0 \pm 1.00$ \\
Ethyl Acetate & $12.0 \pm 1.00$ & $12.33 \pm 0.57$ & $14.0 \pm 1.00$ & $11.0 \pm 1.0$ \\
Chloroform & $11.33 \pm 0.57$ & $9.0 \pm 0.00$ & $10.0 \pm 0.00$ & $11.0 \pm 0.00$ \\
Pet Ether & $11.0 \pm 1.0$ & $12.33 \pm 0.57$ & $11.0 \pm 0.00$ & $11.0 \pm 0.00$ \\
n-Hexane & $11.33 \pm 0.57$ & $9.0 \pm 0.00$ & $12.33 \pm 0.57$ & $12.0 \pm 0.00$ \\
Methanol (Soxhlet) & $13.0 \pm 1.0$ & $14.0 \pm 1.00$ & $11.0 \pm 0.00$ & $11.0 \pm 1.00$ \\
Ethanol (Soxhlet) & $15.33 \pm 0.57$ & $15.0 \pm 1.00$ & $12.33 \pm 0.57$ & $12.0 \pm 1.00$ \\
Ciprofloxacin & $19.0 \pm 1.00$ & $20.0 \pm 1.00$ & $28.0 \pm 1.00$ & $11.0 \pm 0.00$ \\
DMSO & - & - & - & $14.33 \pm 0.57$ \\
\hline
\end{tabular}

['-'-No zone of inhibition was observed] 
Table 2: Zone of inhibition of unripened Anthocephalus cadamba $(\mathrm{mm})$

\begin{tabular}{|c|c|c|c|c|c|}
\hline $\begin{array}{l}\text { Unripened Anthocephalus } \\
\text { Cadamba }\end{array}$ & Staphylococcus aureus & Bacillus cereus & Escherichia coli & $\begin{array}{l}\text { Salmonella } \\
\text { abony }\end{array}$ & Shigella boydii \\
\hline Methanol & $16.0 \pm 1.00$ & $15.33 \pm 0.57$ & $17.0 \pm 1.00$ & $15.0 \pm 1.00$ & $20.0 \pm 0.00$ \\
\hline Ethanol & $12.33 \pm 0.57$ & $14.0 \pm 1.00$ & $11.0 \pm 1.00$ & $12.0 \pm 1.00$ & $18.0 \pm 1.00$ \\
\hline Ethyl Acetate & $14.33 \pm 0.57$ & $14.0 \pm 1.00$ & $15.0 \pm 1.00$ & $13.33 \pm 0.57$ & $18.0 \pm 1.00$ \\
\hline Chloroform & $11.33 \pm 0.57$ & $12.0 \pm 0.00$ & $13.33 \pm 0.57$ & $11.33 \pm 0.57$ & $11.0 \pm 0.00$ \\
\hline Pet Ether & $13.33 \pm 0.57$ & $15.0 \pm 0.00$ & $14.0 \pm 00$ & $13.0 \pm 00$ & $17.00 \pm 0.00$ \\
\hline n-Hexane & $11.0 \pm 1.00$ & $12.00 \pm 0.00$ & - & $12.00 \pm 1.0$ & $11.33 \pm 0.57$ \\
\hline Methanol(Soxhlet) & $14.0 \pm 1.00$ & $14.0 \pm 1.00$ & $14.0 \pm 0.00$ & $14.33 \pm 0.57$ & $19.0 \pm 0.00$ \\
\hline Ethanol (Soxhlet) & $11.0 \pm 0.00$ & $12.0 \pm 0.00$ & 11. $33 \pm 0.57$ & $12.33 \pm 0.57$ & $17.0 \pm 1.00$ \\
\hline Ciprofloxacin & $19.0 \pm 1.00$ & $20.0 \pm 1.00$ & $30.0 \pm 1.00$ & $22.0 \pm 1.00$ & $15.0 \pm 1.00$ \\
\hline DMSO & - & - & - & - & - \\
\hline
\end{tabular}

['-'-No zone of inhibition was observed]

Table 3: Zone of inhibition of Scirpus Kysoor Roxb. (mm)

\begin{tabular}{|c|c|c|c|c|c|}
\hline Scirpus kysoor Roxb. & Staphylococcus aureus & Bacillus Cereus & Escherichia Coli & Salmonellaabony & Shigella boydii \\
\hline Methanol & $14.0 \pm 1.00$ & $12.33 \pm 0.57$ & $12.33 \pm 0.57$ & $13.0 \pm 1.00$ & $23.0 \pm 1.00$ \\
\hline Ethanol & $15.0 \pm 1.00$ & $17.0 \pm 1.00$ & $12.33 \pm 0.57$ & $12.0 \pm 0.00$ & $17.0 \pm 1.00$ \\
\hline Ethyl Acetate & $18.0 \pm 1.00$ & $19.33 \pm 0.57$ & $12.0 \pm 1.00$ & $15.0 \pm 1.00$ & $20.33 \pm 0.57$ \\
\hline Chloroform & $17.33 \pm 0.57$ & $13.0 \pm 0.00$ & $10.0 \pm 0.00$ & $12.0 \pm 1.00$ & $15.0 \pm 1.00$ \\
\hline Pet Ether & $19.33 \pm 0.57$ & $19.33 \pm 0.57$ & $13.0 \pm 1.00$ & $14.0 \pm 0.00$ & $20.33 \pm 0.57$ \\
\hline n-Hexane & $13.0 \pm 0.00$ & $13.0 \pm 0.00$ & $10.0 \pm 0.00$ & $12.0 \pm 1.00$ & $17.0 \pm 0.00$ \\
\hline Ethyl Acetate (Soxhlet) & $13.0 \pm 0.00$ & $17.0 \pm 0.00$ & $11.0 \pm 0.00$ & $10.0 \pm 0.00$ & $20.0 \pm 0.00$ \\
\hline Pet Ether(Soxhlet) & $17.33 \pm 0.57$ & $17.0 \pm 0.00$ & $12.0 \pm 0.00$ & $11.2 \pm 0.00$ & $17.33 \pm 0.57$ \\
\hline Ciprofloxacin & $17.0 \pm 1.00$ & $19.0 \pm 1.00$ & $30.0 \pm 1.00$ & $23.0 \pm 0.00$ & $15.0 \pm 0.00$ \\
\hline DMSO & - & - & - & - & - \\
\hline
\end{tabular}

['-'-No zone of inhibition was observed]

Zone of inhibition $<9 \mathrm{~mm}$ zone was considered as inactive; $9-12 \mathrm{~mm}$ as partially active; while $13-18 \mathrm{~mm}$ as active and $>18 \mathrm{~mm}$ as very active [21]. According to this, most potent extracts of all plant materials were found to be either active or very active. Among the different solvent extracts studied, for ripened Anthocephalus cadamba fruits, macerated ethanol extract was found to be most potent extract against all the pathogens whereas for unripened fruits macerated methanol extract was found to be most potent. In the case of Scirpus kysoor Roxb. tuber macerated ethyl acetate and pet ether extracts were found to be most potent against all the pathogens. The maximum zone of inhibition was observed against $S$. boydii by macerated ethanol extract of ripened Anthocephalus cadamba fruit which was $24 \mathrm{~mm}$. (shown in fig. 1). For S. Aureus highest antimicrobial activity exhibited by macerated pet ether extract of Scirpus kysoor Roxb. tuber $(19 \mathrm{~mm})$ followed by macerated ethanol extract (18 mm) of ripened Anthocephalus cadamba. For $B$. cereus macerated Pet ether and ethyl acetate extract of Scirpus kysoor Roxb. tuber (19 $\mathrm{mm}$ ) followed by macerated ethanol extract (18 $\mathrm{mm}$ ) of ripened Anthocephalus cadamba showed the highest activity. For E. coli, S. abony and S. boydii macerated ethanol extract of ripened Anthocephalus cadamba showed the highest activity (19, 17, $24 \mathrm{~mm}$ resp).

In general, ripened Anthocephalus cadamba showed greater antimicrobial activity as compared to unripened Anthocephalus cadamba.

The activity of potent extracts against all the pathogens (except $E$. coli) were found to be comparable with that of positive control.

Both soxhlets, as well as macerated extracts, showed antimicrobial activity but in most of the cases for same solvent and concentration, the zone of inhibition was found to be greater for macerated extracts.

Thus, all the plant extracts tested in the present study displayed broad spectrum antibacterial activity (that is against both gram positive and gram negative type of bacteria). However, differences observed between antibacterial activities of the extracts could be due to the differences in the chemical composition (i.e. different bioactive phytochemical groups/metabolites) of these extracts. Such secondary metabolites of plants have been reported to have many effects including antibacterial and antiviral properties [22, 23].

\section{Determination of MIC and MBC}

The antibacterial activity of the plant extracts is depicted in table 6-8. The results indicated that the plant extracts showed antibacterial activities at variable degrees against all the pathogens, with MIC values varying from 0.312 to $25.0 \mathrm{mg} / \mathrm{ml}$. The lowest value of MIC was observed for macerated ethyl acetate extract of Scirpus kysoor Roxb. against B. cereus $(0.312 \mathrm{mg} / \mathrm{ml})$. It is shown in fig. 2.

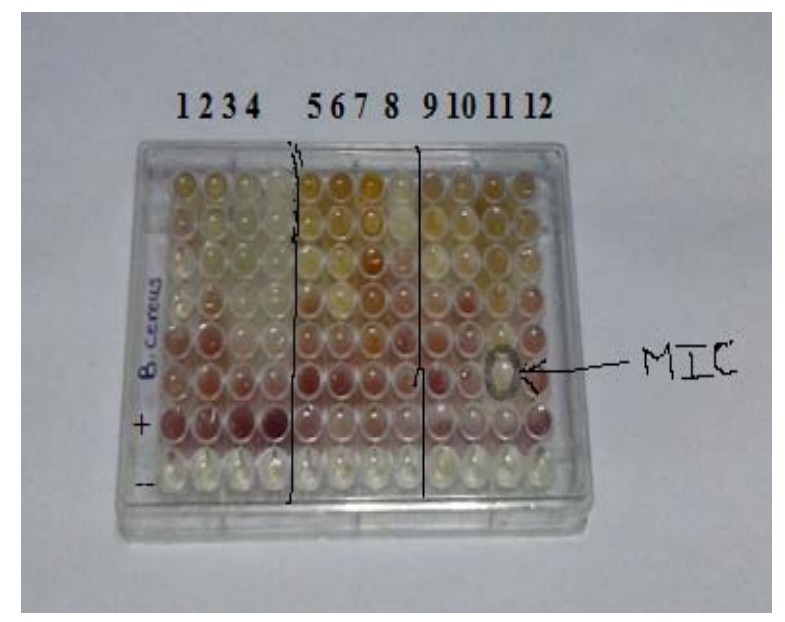

Fig. 2: MIC of plant materials against bacillus cereus, 1-4, 58, 9-12:-methanol,ethanol,ethyl acetate and pet ether extracts of unripened Anthocephalus cadamba, ripened Anthocephalus cadamba and Scirpus kysoor Roxb. Respectively 
Table 4: MIC, MBC and MBC: MIC ratio of ripened Anthocephalus Cadamba

\begin{tabular}{|c|c|c|c|c|c|c|c|c|c|c|c|c|c|c|c|}
\hline \multirow{2}{*}{$\begin{array}{l}\text { Ripened } \\
\text { Cadamba } \\
\text { Solvent }\end{array}$} & \multicolumn{3}{|c|}{$\begin{array}{l}\text { Staphylococcus } \\
\text { aureus }\end{array}$} & \multicolumn{3}{|c|}{ Bacillus cereus } & \multicolumn{3}{|c|}{ Escherichia coli } & \multicolumn{3}{|c|}{ Salmonella abony } & \multicolumn{3}{|c|}{ Shigella boydii } \\
\hline & $\begin{array}{l}\text { MIC } \\
\text { (mg/ } \\
\mathrm{ml})\end{array}$ & $\begin{array}{l}\text { MBC } \\
(\mathrm{mg} \\
/ \mathrm{ml})\end{array}$ & $\begin{array}{l}M B C: \\
M I C\end{array}$ & $\begin{array}{l}\text { MIC } \\
\text { (mg/ } \\
\mathrm{ml})\end{array}$ & $\begin{array}{l}\text { MBC } \\
\text { (mg/ } \\
\mathrm{ml})\end{array}$ & $\begin{array}{l}\text { MB } \\
\text { C: } \\
\text { MIC }\end{array}$ & $\begin{array}{l}\text { MIC } \\
\text { (mg/ } \\
\mathrm{ml})\end{array}$ & $\begin{array}{l}\text { MBC } \\
\text { (mg/ } \\
\text { ml) }\end{array}$ & $\begin{array}{l}\text { MBC: } \\
\text { MIC }\end{array}$ & $\begin{array}{l}\text { MIC } \\
\text { (mg/ } \\
\mathrm{ml})\end{array}$ & $\begin{array}{l}\text { MBC } \\
\text { (mg/ } \\
\mathrm{ml})\end{array}$ & $\begin{array}{l}\text { MB } \\
\text { C: } \\
\text { MIC }\end{array}$ & $\begin{array}{l}\text { MIC } \\
\text { (mg/ } \\
\mathrm{ml})\end{array}$ & $\begin{array}{l}\text { MBC } \\
\text { (mg/ } \\
\mathrm{ml})\end{array}$ & $\begin{array}{l}\text { MB } \\
\text { C: } \\
\text { MIC }\end{array}$ \\
\hline Methanol & 6.25 & 6.25 & 1 & 6.25 & 25.0 & 4 & 12.5 & 25.0 & 2 & 12.5 & 12.5 & 1 & 6.25 & 25.0 & 4 \\
\hline Ethanol & 3.12 & 3.12 & 1 & 3.12 & 3.12 & 1 & 12.5 & $\begin{array}{l}\text { Above } \\
25.0\end{array}$ & - & 12.5 & $\begin{array}{l}\text { Above } \\
25.0\end{array}$ & - & 6.25 & 25.0 & 4 \\
\hline $\begin{array}{l}\text { Ethyl } \\
\text { Acetate }\end{array}$ & 6.25 & 6.25 & 1 & 12.5 & 12.5 & 1 & 25.0 & 25.0 & 1 & 12.5 & $\begin{array}{l}\text { Above } \\
25.0\end{array}$ & - & 12.5 & 25.0 & 2 \\
\hline Pet Ether & 12.5 & 12.5 & 1 & 12.5 & 12.5 & 1 & 25.0 & $\begin{array}{l}\text { Above } \\
25.0\end{array}$ & - & NA & NA & NA & 12.5 & 25.0 & 2 \\
\hline n-Hexane & NA & NA & NA & NA & NA & NA & NA & NA & NA & 12.5 & 12.5 & 1 & NA & NA & NA \\
\hline $\begin{array}{l}\text { Methanol } \\
\text { (Soxhlet) }\end{array}$ & 12.5 & 12.5 & 1 & 6.25 & 6.25 & 1 & 12.5 & $\begin{array}{l}\text { Above } \\
25.0\end{array}$ & - & 6.25 & $\begin{array}{l}\text { Above } \\
25.0\end{array}$ & - & 6.25 & - & - \\
\hline $\begin{array}{l}\text { Ethanol } \\
\text { (Soxhlet) }\end{array}$ & 6.25 & $\begin{array}{l}\text { Abov } \\
\text { e } \\
25.0\end{array}$ & - & 3.12 & 12.5 & 4 & 12.5 & $\begin{array}{l}\text { Above } \\
25.0\end{array}$ & - & 6.25 & $\begin{array}{l}\text { Above } \\
25.0\end{array}$ & - & 6.25 & - & - \\
\hline
\end{tabular}

['-'-not calculated, NA-not applicable]

Table 5: MIC, MBC and MBC: MIC ratio of Unripened Anthocephalus Cadamba

\begin{tabular}{|c|c|c|c|c|c|c|c|c|c|c|c|c|c|c|c|}
\hline \multirow{2}{*}{$\begin{array}{l}\text { Unripened } \\
\text { Cadamba } \\
\text { Solvent }\end{array}$} & \multicolumn{3}{|c|}{$\begin{array}{l}\text { Staphylococcus } \\
\text { aureus }\end{array}$} & \multicolumn{3}{|c|}{ Bacillus cereus } & \multicolumn{3}{|c|}{ Escherichia coli } & \multicolumn{3}{|c|}{ Salmonella abony } & \multicolumn{3}{|c|}{ Shigella boydii } \\
\hline & $\begin{array}{l}\text { MIC } \\
\text { (mg/ } \\
\mathrm{ml})\end{array}$ & $\begin{array}{l}\text { MBC } \\
(\mathrm{mg} \\
/ \mathrm{ml})\end{array}$ & $\begin{array}{l}\text { MB } \\
\text { C: } \\
\text { MIC }\end{array}$ & $\begin{array}{l}\text { MIC } \\
\text { (mg/ } \\
\mathrm{ml})\end{array}$ & $\begin{array}{l}\text { MBC } \\
\text { (mg/ } \\
\mathrm{ml})\end{array}$ & $\begin{array}{l}\text { MB } \\
\text { C: } \\
\text { MIC }\end{array}$ & $\begin{array}{l}\text { MIC } \\
\text { (mg/ } \\
\mathrm{ml})\end{array}$ & $\begin{array}{l}\text { MBC } \\
\text { (mg/ } \\
\mathrm{ml})\end{array}$ & $\begin{array}{l}\text { MB } \\
\text { C: } \\
\text { MIC }\end{array}$ & $\begin{array}{l}\text { MIC } \\
\text { (mg/ } \\
\mathrm{ml})\end{array}$ & $\begin{array}{l}\text { MBC } \\
\text { (mg/ } \\
\mathrm{ml})\end{array}$ & $\begin{array}{l}\text { MB } \\
\text { C: } \\
\text { MIC }\end{array}$ & $\begin{array}{l}\text { MIC } \\
\text { (mg/ } \\
\mathrm{ml})\end{array}$ & $\begin{array}{l}\text { MBC } \\
\text { (mg/ } \\
\mathrm{ml})\end{array}$ & $\begin{array}{l}\text { MB } \\
\text { C: } \\
\text { MIC }\end{array}$ \\
\hline Methanol & 6.25 & 6.25 & 1 & 3.15 & 6.25 & 2 & 12.5 & $\begin{array}{l}\text { Above } \\
25.0\end{array}$ & - & 12.5 & $\begin{array}{l}\text { Above } \\
25.0\end{array}$ & - & 12.5 & 25.0 & 2 \\
\hline Ethanol & 6.25 & 12.5 & 2 & 6.25 & 6.25 & 1 & 12.5 & $\begin{array}{l}\text { Above } \\
25.0\end{array}$ & - & 12.5 & $\begin{array}{l}\text { Above } \\
25.0\end{array}$ & - & 12.5 & 25.0 & 2 \\
\hline $\begin{array}{l}\text { Ethyl } \\
\text { Acetate }\end{array}$ & 1.56 & 3.12 & 2 & 1.56 & 6.25 & 4 & 12.5 & $\begin{array}{l}\text { Above } \\
25.0\end{array}$ & - & 12.5 & $\begin{array}{l}\text { Above } \\
25.0\end{array}$ & - & 6.25 & 25.0 & 4 \\
\hline Pet Ether & 1.56 & 3.12 & 2 & 1.56 & 6.25 & 4 & 25.0 & $\begin{array}{l}\text { Above } \\
25.0\end{array}$ & - & 12.5 & $\begin{array}{l}\text { Above } \\
25.0\end{array}$ & - & 6.25 & 25.0 & 4 \\
\hline $\begin{array}{l}\text { Methanol } \\
\text { (Soxhlet) }\end{array}$ & 12.5 & $\begin{array}{l}\text { Abov } \\
\text { e } \\
25.0\end{array}$ & - & 6.25 & $\begin{array}{l}\text { Above } \\
25.0\end{array}$ & - & 25.0 & $\begin{array}{l}\text { Above } \\
25.0\end{array}$ & - & 12.5 & $\begin{array}{l}\text { Above } \\
25.0\end{array}$ & - & 12.5 & $\begin{array}{l}\text { Above } \\
25.0\end{array}$ & - \\
\hline $\begin{array}{l}\text { Ethyl } \\
\text { Acetate } \\
\text { (Soxhlet) }\end{array}$ & 3.12 & $\begin{array}{l}\text { Abov } \\
\text { e } \\
25.0\end{array}$ & - & 3.12 & $\begin{array}{l}\text { Above } \\
25.0\end{array}$ & - & 12.5 & $\begin{array}{l}\text { Above } \\
25.0\end{array}$ & - & 12.5 & $\begin{array}{l}\text { Above } \\
25.0\end{array}$ & - & 12.5 & $\begin{array}{l}\text { Above } \\
25.0\end{array}$ & - \\
\hline
\end{tabular}

['-'-not calculated, NA-not applicable]

Table 6: MIC, MBC and MBC: MIC ratio of Scirpus Kysoor Roxb

\begin{tabular}{|c|c|c|c|c|c|c|c|c|c|c|c|c|c|c|c|}
\hline \multirow{2}{*}{$\begin{array}{l}\text { Scirpus } \\
\text { Kysoor Roxb. } \\
\text { Solvent }\end{array}$} & \multicolumn{3}{|c|}{$\begin{array}{l}\text { Staphylococcus } \\
\text { aureus }\end{array}$} & \multicolumn{3}{|c|}{ Bacillus cerius } & \multicolumn{3}{|c|}{ Escherichia coli } & \multicolumn{3}{|c|}{ Salmonella abony } & \multicolumn{3}{|c|}{ Shigella boydii } \\
\hline & $\begin{array}{l}\text { MIC } \\
\text { (mg/ } \\
\mathrm{ml}) \\
\end{array}$ & $\begin{array}{l}\text { MBC } \\
\text { (mg/ } \\
\mathrm{ml})\end{array}$ & $\begin{array}{l}\text { MB } \\
\text { C: } \\
\text { MIC } \\
\end{array}$ & $\begin{array}{l}\text { MIC } \\
\text { (mg/ } \\
\mathrm{ml})\end{array}$ & $\begin{array}{l}\text { MBC } \\
\text { (mg/ } \\
\mathrm{ml})\end{array}$ & $\begin{array}{l}\text { MB } \\
\text { C: } \\
\text { MIC }\end{array}$ & $\begin{array}{l}\text { MIC } \\
(\mathrm{mg} \\
/ \mathrm{ml})\end{array}$ & $\begin{array}{l}\text { MBC } \\
(\mathrm{mg} / \\
\mathrm{ml})\end{array}$ & $\begin{array}{l}\text { MB } \\
\text { C: } \\
\text { MIC } \\
\end{array}$ & $\begin{array}{l}\text { MIC } \\
\text { (mg/ } \\
\mathrm{ml})\end{array}$ & $\begin{array}{l}\text { MBC } \\
\text { (mg/ } \\
\mathrm{ml})\end{array}$ & $\begin{array}{l}\text { MB } \\
\text { C: } \\
\text { MIC } \\
\end{array}$ & $\begin{array}{l}\text { MIC } \\
(\mathrm{mg} / \\
\mathrm{ml})\end{array}$ & $\begin{array}{l}\text { MBC } \\
\text { (mg/ } \\
\mathrm{ml})\end{array}$ & $\begin{array}{l}\text { MBC } \\
: \\
\text { MIC } \\
\end{array}$ \\
\hline Methanol & NA & NA & NA & 2.5 & 2.5 & 1 & 10.0 & $\begin{array}{l}\text { Above } \\
10.0\end{array}$ & - & 5.0 & 10.0 & 2 & 5.0 & 10.0 & 2 \\
\hline Ethanol & 1.25 & 1.25 & 1 & 5.0 & 5.0 & 1 & 5.0 & $\begin{array}{l}\text { Above } \\
10.0\end{array}$ & - & 5.0 & 10.0 & 2 & 5.0 & 10.0 & 2 \\
\hline Ethyl Acetate & 0.62 & 0.62 & 1 & 0.31 & 0.31 & 1 & 5.0 & $\begin{array}{l}\text { Above } \\
10.0\end{array}$ & - & 5.0 & $\begin{array}{l}\text { Above } \\
10.0\end{array}$ & - & 1.25 & 10.0 & 8 \\
\hline Chloroform & 0.62 & 2.5 & 4 & NA & NA & NA & NA & NA & NA & NA & NA & NA & NA & NA & NA \\
\hline Pet Ether & 1.25 & 1.25 & 1 & 1.25 & 5.0 & 4 & 10.0 & $\begin{array}{l}\text { Above } \\
10.0\end{array}$ & - & 5.0 & $\begin{array}{l}\text { Above } \\
10.0\end{array}$ & - & 5.0 & 10.0 & 2 \\
\hline $\begin{array}{l}\text { EthylAcetate } \\
\text { (Soxhlet) }\end{array}$ & 1.25 & $\begin{array}{l}\text { Abov } \\
\text { e } \\
10.0\end{array}$ & - & 1.25 & 2.50 & 2 & 5.0 & $\begin{array}{l}\text { Above } \\
10.0\end{array}$ & - & 5.0 & $\begin{array}{l}\text { Above } \\
10.0\end{array}$ & - & 2.5 & $\begin{array}{l}\text { Abov } \\
\text { e } \\
10.0\end{array}$ & - \\
\hline $\begin{array}{l}\text { Pet Ether } \\
\text { (Soxhlet) }\end{array}$ & 1.25 & 2.5 & 4 & 1.25 & 2.50 & 2 & 5.0 & $\begin{array}{l}\text { Above } \\
10.0\end{array}$ & - & 2.5 & $\begin{array}{l}\text { Above } \\
10.0\end{array}$ & - & 2.5 & $\begin{array}{l}\text { Abov } \\
\mathrm{e} \\
10.0 \\
\end{array}$ & - \\
\hline
\end{tabular}

['-'-not calculated, NA-not applicable] 
In the case of Ripened Anthocephalus cadamba fruit, the lowest value of MIC was shown by macerated as well as soxhlet extract of ethanol against all the pathogens whereas in the case of unripened Anthocephalus cadamba; lowest MIC value was shown by macerated ethyl acetate and pet ether extracts. Macerated Ethyl acetate extract of Scirpus kysoor Roxb. exhibited lowest MIC values against all the pathogens. For $S$. Aureus,B. Cereus and S. boydii lowest MIC was shown by macerated Ethyl acetate extract of Scirpus kysoor Roxb. $(0.625 \mathrm{mg} / \mathrm{ml}, 0.312 \mathrm{mg} / \mathrm{ml}$ and $1.25 \mathrm{mg} / \mathrm{ml}$ respectively.) whereas For E. coli and S. abony also Lowest MIC was $5.0 \mathrm{mg} / \mathrm{ml}$.)Thus, all these plant materials were found to be a more efficient antimicrobial agent against $S$. Aureus, B. Cereus and S. boydii than that of E. Coli and $S$. Abony on the basis of their MIC. Most of these extracts were found to be bactericidal (exception: macerated ethyl acetate extract of Scirpus kysoor Roxb. Against $S$. boydii) showing ratio of $\mathrm{MBC}$ : $\mathrm{MIC}</=4$, against $S$. Aureus, B. Cereus and $S$. boydii whereas for rest 2 pathogens they were considered as bacteriostatic as MBC: MIC $>4$ [24].

Macerated extract showed lowest MIC as compared to soxhlet extracts of the same solvent of same concentration for $S$. Aureus, $B$. Cereus and $S$. boydii whereas it was found to be same for rest 2 pathogens with few exceptions. Both the soxhlet extracts of ripened Anthocephalus cadamba fruit and Scirpus kysoor Roxb. tuber were found to be bactericidal against B. Cereus. Also, soxhlet methanol extract of ripened Anthocephalus cadamba and soxhlet pet ether extract of Scirpus kysoor Roxb. were found to be bactericidal against S. Aureus. Rest soxhlet extracts were observed as bacteriostatic against remaining 3 pathogens.

The results for antibacterial activity of Anthocephalus cadamba were found to be comparable to those obtained by other authors. They reported that ethanolic extract of Anthocephalus cadamba showed significant antibacterial activity against Gram positive and Gram negative organisms. Alcoholic extract of fruits of Anthocephalus cadamba showed significant antibacterial activity against E. coli, $S$. aureus with the zone of inhibition of $22-24 \mathrm{~cm}$ and low MIC value up to $1.00 \mathrm{mg} / \mathrm{ml}[5,7]$. The difference observed in some results may be due to the difference in the type of pathogenic strains used and method of extraction used in the present study.

From the zone of inhibition and MIC determination, it was observed that zones of inhibition in agar diffusion assay do not necessarily correlate to MIC values in the broth microdilution method,as already stated by Ncube et al.(2008) [25].

The results of the present work also bring additional data on the antibacterial activity of ripened and unripened Anthocephalus cadamba fruit, since we report for the first time its activity against $B$. cereus,S. abony and S. boydii. MBC is also reported first time for all the pathogens. Also, to best of our knowledge antimicrobial activity against all the pathogens for Scirpus kysoor Roxb. is described here for the first time.

\section{CONCLUSION}

From the present investigation, we can conclude that all the 3 plant materials contain potential antimicrobial components that may be useful for the development of pharmaceutical industries as a broad spectrum antibiotic against various diseases caused by food pathogens. The results of the study support the development of new antimicrobial drugs from both the plants. After this screening experiment, phytochemical studies and bioautographic studies of potent extracts will be necessary to isolate the active constituents and evaluate the antibacterial activities against selected food pathogens.

\section{CONFLICT OF INTERESTS}

Declare none

\section{REFERENCES}

1. Mendel Friedman, Philip R Henika, Robert E Mandrell. Bactericidal activities of plant essential oils and some of their isolated constituents against Campylobacter jejuni, Escherichia coli, Listeria monocytogenes, and Salmonella enteri. J Food Prot 2002;68:1545-60.
2. Antara Sen, Amlan Batra. Evaluation of antimicrobial activity of different solvent extracts of medicinal plant: Melia Azedarach L. Int J Curr Pharm Res 2012;4:67-73.

3. Bin Shan, Yi-Zhong Cai, John D, Brooks, Horold Corke. Antibacterial properties and major bioactive components of cinnamon stick (Cinnamomumburmannii): Activity against foodborne pathogenic bacteria. J Agric Food Chem 2007;55:5484-90.

4. Dubey A, Nayak S, Goupale DC. A review on phytochemical, pharmacological and toxicological studies. Pharm Lett 2011;3:45-54.

5. Mishra RP. Antibacterial properties of Anthocephalus cadamba Fruits. Webmed Central Ayurvedic Med 2011;2:WMC002073.

6. Mishra RP. A comparative study and extract optimization for antimicrobial properties of different parts of Anthocephalus cadamba. Webmed Central Ayurvedic Med 2013;4:WMC002116.

7. Sandhya I, Durve A. Isolation and characterization of the antimicrobial compound from fruits of AnthocephalusIndicusA. Rich. Int J Pharm Pharm Sci 2014;6:285-91.

8. Umachigi SP, Kumar GS, Jayaveera K, Kishore KDV, Ashok KCK, Dhanapal R. Antimicrobial, wound healing and antioxidant activities of Anthocephalus cadamba. African journal of traditional, complementary, and alternative medicines. Afr Networks Ethnomed 2007;4:481-7.

9. The Ayurvedic Pharmacopoeia of India. (Part-1, Vol-1). The government of India (Ministry of Health and family welfare); 2007. p. 92-3.

10. Brahmshankar Mishra, Rupalalji Vaishya. Bhavaprakash Nighantu. (part-1). Chaikhamba Sanskrit Sansthan, Varanasi; 1990. p. 701-2.

11. Handa SS. An overview of extraction techniques for medicinal and aromatic plants. In: Handa SS, Khanuja S, Longo G, Rakesh DD. Editors. Extraction technologies for medicinal and aromatic plants Trieste (Italy): International Centre for Science and high Technology (ICS-UNIDO); 2008. p. 21-52.

12. K Das, RKS Tiwari, DK Shrivastava. Techniques for evaluation of medicinal plant products as antimicrobial agent: current methods and future trends. J Med Plants Res 2010;4:104-11.

13. Performance Standards For Antimicrobial Susceptibility Testing. 21st Informational Supplement by Clinical and Laboratory Standards Institute (CLSI), Document M100-S21; 2011. p. 30.

14. Irshad S, Maryum M, Farzana P. In vitro antibacterial activities of three medicinal plants using agar well diffusion method. Res J Biol 2012;2:1-8.

15. Andrews JM. Determination of minimum inhibitory concentration. J Antimicrob Chemother 2001;48:5-16.

16. Thongson C, Davidson PM, Mahakarrchanakul W, Weiss J. Antimicrobial activity of ultrasound-associated solvent extracted species. Lett Appl Microbiol 2004;39:401-6.

17. Methods For Dilution Antimicrobial Susceptibility Tests For Bacteria That Grow Aerobically; Approved Standard-Seventh Edition. By Clinical and Laboratory Standard Institute (CLSI). Document M7-A7; 2006. p. 26.

18. Wesler A, Geiss HK, Saller R, Reichiling J. A novel colorimetric broth microdilution method to determine minimum inhibitory concentration (MIC) of antibiotics and essential oil against helicobacter pyroli. Pharmazie 2005;60:498-502.

19. Lagnika L, Anago E, Atindehou M, Atindehou M, Adjahoutonon B, Dramane K, et al. Antimicrobial activity of Crataeva Religiosa forst against bacteria isolated from thryonomysswinderianus temmink. Afr J Biotechnol 2011;10:10034-9.

20. Andrews JM. Determination of minimum inhibitory concentrations. J Antimicrob Chemother 2001;48(S1);S-16.

21. Pundir RK, Jain P, Sharma C. Antimicrobial activity of ethanolic extracts of Syzygiumaromaticum and Alliumsativum against foodassociated bacteria and fungi. Ethnobotanical Leaflets 2010:14:344-60.

22. Noumedem J, Mihasan M, Lacmata S, Stefan M, Kuiate J, Kuete V. Antibacterial activities of the methanol extracts of ten cameroonian vegetables against gram-negative multidrugresistant bacteria. BMC Complementary Altern Med 2013;13:26.

23. Cowan MM. Plant products as antimicrobial agents. Clin Microbiol Rev 1999;12:564-82. 
24. Djeussi DE, Noumedem JA, Seukep JA, Fankam AG, Voukeng IK, Tankeo SB, et al. Antibacterial activities of selected edible plants extracts against multidrug-resistant Gramnegative bacteria. BMC Complementary Altern Med 2013; 13:164.

25. Ncube NS, Afolayan AJ, Okoh AI. Assessment techniques of antimicrobial properties of natural compounds of plant origin: current methods and future trends. Afr J Biotechnol 2008;7:1797-806.

\section{How to cite this article}

- Hemangi Datar, Ajit Datar. Antimicrobial activity of Anthoceplalus cadamba and Scirpus kysoor roxb. against food pathogens. Int J Curr Pharm Res 2016;8(4):13-18. 